\title{
Surgical Site Infections Caused by Highly Virulent Methicillin-Resistant Staphylococcus aureus Sequence Type 398, China
}

\author{
Lu Sun, ${ }^{1}$ Yan Chen, ${ }^{1}$ Danying Wang, \\ Haiping Wang, Dandan Wu, \\ Keren Shi, Ping Yan, Yunsong Yu
}

We identified 2 methicillin-resistant Staphylococcus aureus strains of sequence type 398 from surgical site infections in China. Genetic analysis and clinical data from these strains suggested that they were human-related but sporadic. Hemolysis analysis and mouse-skin infection models indicated a high virulence potential for these strains.

$\mathrm{M}$ ethicillin-resistant Staphylococcus aureus (MRSA) can cause a variety of infections, such as skin and soft tissue infection (SSTI), pneumonia, and sepsis. MRSA can also cause surgical site infections (SSI), which are the most common type of healthcare-associated infections in hospitals ( 1 ). In addition, MRSA infection has been reported in animals, including pigs, veal calves, and poultry (2). Livestock-associated MRSA (LA-MRSA) was first reported in humans in the Netherlands in 2003 (3), and numerous countries have subsequently reported the presence of this MRSA variant. Although LA-MRSA belongs to several clone complexes, the major clone of LA-MRSA belongs to sequence type (ST) 398 (4).

Several infections caused by MRSA ST398 have been reported in the community, ranging from mild skin infections to serious invasive infections, both with and without livestock contact. Fatal cases of septicemia were also reported in Japan and Denmark (5,6). MRSA ST398 isolates from human postoperative surgical site infections were presented with complete genome sequencing in Canada and in a long-term MRSA surveillance study in the United Kingdom $(7,8)$. The virulence potential of MRSA ST398 isolated from SSI has not been reported to date.

Author affiliations: Sir Run Run Shaw Hospital of Zhejiang University School of Medicine, Hangzhou, China (L. Sun, Y. Chen, D. Wang, H. Wang, D. Wu, K. Shi, Y. Yu); Fuyang Hospital of Traumatology and Orthopedics of Traditional Chinese Medicine, Hangzhou (P. Yan); Key Laboratory for Microbial Technology and Bioinformatics of Zhejiang Province, Hangzhou (Y. Yu)

DOI: https://doi.org/10.3201/eid2501.171862
We studied 2 MRSA ST398 strains from surgical site infections, focusing on their clinical characteristics, genetic features, and virulence potential. The study was approved by the local ethics committees in Sir Run Run Shaw Hospital with a waiver of informed consent (approval no. 20150115-1). Mouse experiments were performed with approval from the Institutional Animal Ethics Committee of Zhejiang University (approval no. ZJU2015-141-01).

\section{The Study}

From January 2013 through December 2014, we collected a total of 147 Staphylococcus aureus isolates at Fuyang Hospital of Traumatology and Orthopedics of Traditional Chinese Medicine, a tertiary hospital in Hangzhou, China, with $\approx 500$ beds. We performed multilocus sequence type (MLST) analysis on 18 SSI MRSA isolates from patients who underwent orthopedic surgery. We detected 2 MRSA strains, FY20 and FY22, which belong to ST398.

We characterized these strains by antimicrobial drug susceptibility testing, whole-genome sequencing and comparison, hemolysis analysis, and a mouse skin infection model. We included 2 community-acquired MRSA (CAMRSA) strains isolated from patients in 2015 at Sir Run Run Shaw Hospital for comparison; SR389 was isolated from a 32-year-old female outpatient with a left neck mass, and SR411 was isolated from a 21-year-old female outpatient with lower extremity nodules and an ulcer. We sequenced the genomes on an Illumina HiSeq2000 platform (Illumina, http://www.illumina.com). We deposited the draft genome of FY20 as GenBank accession no. NTMC00000000, for FY22 as NXFU00000000, for SR389 as PDFA00000000, and for SR411 as PDFB00000000. We imported FASTA files into SeqSphere+ software version 4.1 (Ridom GmbH, http://www.ridom.de/seqsphere) for analysis (9). We detected virulence and resistance genes using the Center for Genomic Epidemiology website (http://www.genomicepidemiology.org).

We collected supernatants from bacterial cultures grown at $37^{\circ} \mathrm{C}$ for $24 \mathrm{~h}$ with shaking at $180 \mathrm{rpm}$. We determined hemolytic activities by incubating samples with human red blood cells ( $2 \% \mathrm{vol} / \mathrm{vol}$ in Dulbecco's phosphate-buffered saline) for $1 \mathrm{~h}$ at $37^{\circ} \mathrm{C}$ and subsequently by

${ }^{1}$ These authors contributed equally to this article. 
Table. Characteristics of methicillin-resistant Staphylococcus aureus sequence type 398 isolates from 4 patients, China*

\begin{tabular}{|c|c|c|c|c|}
\hline \multirow[b]{2}{*}{ Characteristic } & \multicolumn{4}{|c|}{ Isolate } \\
\hline & FY20 & FY22 & SR389 & SR411 \\
\hline Patient age, y/sex & $42 / \mathrm{F}$ & $52 / \mathrm{M}$ & $32 / \mathrm{F}$ & $21 / \mathrm{F}$ \\
\hline Admission diagnosis & $\begin{array}{l}\text { Right tibial plateau } \\
\text { comminuted fracture }\end{array}$ & $\begin{array}{l}\text { Right tibial plateau } \\
\text { comminuted fracture }\end{array}$ & Left neck mass & $\begin{array}{l}\text { Lower extremity nodules } \\
\text { with ulceration }\end{array}$ \\
\hline Infection type & SSI & SSI & SSTI & SSTI \\
\hline SCCmec type & V & V & V & V \\
\hline spa type & t034 & t034 & t034 & $\mathrm{t} 1255$ \\
\hline Panton-Valentine leukocidin & - & - & - & - \\
\hline Resistance phenotype & PEN, OXA, CLI, ERY & PEN, OXA & PEN, OXA & PEN, OXA, CLI, ERY \\
\hline Resistance genes & mecA, blaZ, ermC & mecA, blaZ & $\operatorname{mec} A$, blaZ & mecA, blaZ, ermC \\
\hline Virulence factors & $\begin{array}{c}\text { aur, sak, scn, hlb, hlgA, } \\
\text { hlgB, hlgC }\end{array}$ & $\begin{array}{c}\text { aur, sak, scn, hlb, hlgA, } \\
\text { hlgB, hlgC }\end{array}$ & $\begin{array}{c}\text { aur, sak, scn, hlb, hlgA, } \\
\text { hlgB, hlgC }\end{array}$ & $\begin{array}{c}\text { aur, sak, scn, hlb, hlgA, } \\
\text { hlgB, hlgC }\end{array}$ \\
\hline
\end{tabular}

measuring the optical density at $540 \mathrm{~nm}$ using an ELISA reader. We performed the mouse skin infection model using BALB/c female nude mice, 4-6 weeks of age. We injected anesthetized mice subcutaneously with $\approx 1 \times 10^{7}$ bacterial cells in $50 \mu \mathrm{L}$ phosphate-buffered saline in the back. We measured length and width of the abscess or lesion for 6 days with calipers, and calculated abscess size by length $\times$ width. We euthanized all mice 6 days after injection. We used South Korea CA-MRSA strain HL1 (ST72) and its mutant HL1 $\triangle a g r$ in the skin infection model and the hemolysis study for comparison (10). We performed statistical analysis using GraphPad Prism version 7.01 (GraphPad Software, https://www.graphpad.com).

We isolated both MRSA strains from SSIs, FY20 and FY22. FY20 was isolated from a 42-year-old female patient who had undergone steel plate-screw internal fixation after a right tibial plateau comminuted fracture in May 2014. She was discharged in June 2014 and admitted to the hospital again because of swelling and pain in her right knee 71 days after surgery. The abscess was surgically

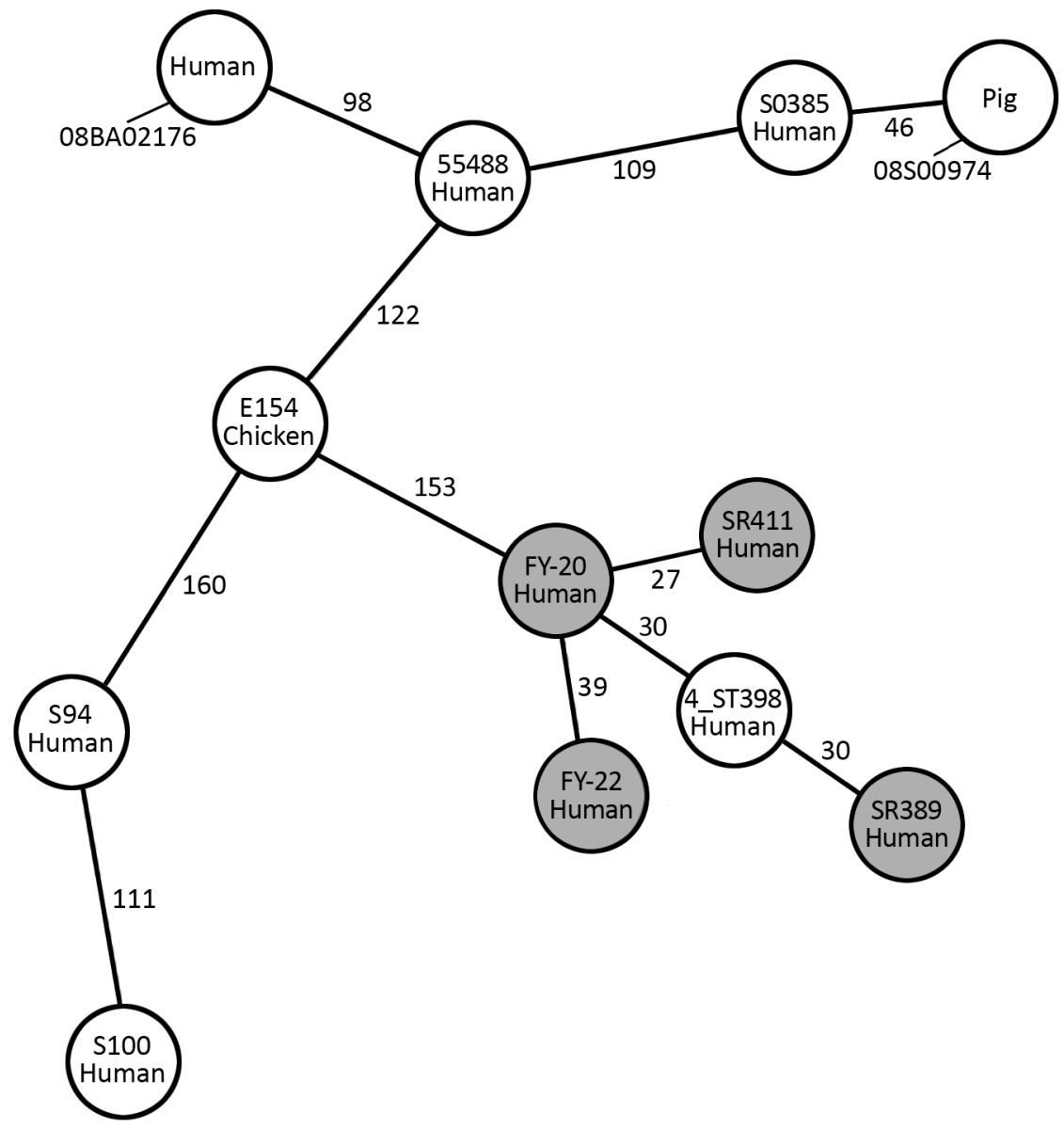

Figure 1. Minimum-spanning tree built from the core genome multilocus sequence type allelic profiles of MRSA ST398 strains from 4 patients in China (gray circles) and other ST398 strains. Each circle represents a single strain and is named with the sample and the origin. The 12 strains are based on 1,807 columns with the pairwise ignoring missing values option in Ridom SeqSphere+ software (Ridom GmbH, http://www. ridom.de/seqsphere). The numbers on the connecting lines indicate the number of allelic differences between 2 strains. $S$. aureus strain COL (GenBank accession no. NC_002951) is used as a reference. S0385 (human, MRSA, NC_017333.1), 08BA02176 (human, MRSA, CP003808.1), 55488 (human, MRSA, NZ_LAWV00000000), 4_ST398 (human, MRSA, He L et al, 2018), S94 (human, MSSA, AUPW00000000), S100 (human, MSSA, AUPV00000000), 08S00974 (animal, MRSA, NZ_CP020019.1), and E154 (animal, MRSA, CP013218.1), are used for comparison. MRSA, methicillinresistant Staphylococcus aureus; ST, sequence type. 
debrided, and she was treated with levofloxacin. We isolated FY22 from a 52-year-old male patient. This patient also underwent steel plate-screw internal fixation after a right tibial plateau comminuted fracture in August 2014. He was discharged in September 2014 and was admitted to the hospital again for pus and exudate from the incision site in the right knee 21 days after surgery. The wound was surgically debrided, and he was treated with cefuroxime and levofloxacin. These 2 patients were admitted to different wards and were treated by different teams during hospitalization.

Antimicrobial susceptibility testing results showed that the 4 ST398 strains in our study had a similar resistance phenotype; they displayed resistance to $\beta$-lactam antimicrobial drugs but were susceptible to most other antimicrobial drugs. FY20 and SR411 were resistant to clindamycin and erythromycin. Resistance genes detected using the Center for Genomic Epidemiology site matched the resistance phenotypes (Table). The 4 ST398 strains were negative for tet $M$ and $c z r C$, were positive for $\varphi S a 3$, and displayed susceptibility to tetracycline. We classified FY20, FY22, and SR389 into SCCmec V and spa-type t034, and SR411 into SCCmec V and spa-type t1255. We built a minimum-spanning tree based on the core genome MLST (cgMLST) allelic profiles (Figure 1). There were 27 allelic differences by cgMLST analysis between FY20 and SR411; for 5 pairs of other strains from our study (FY20 and FY22, FY20 and SR389, FY22 and SR389, FY22 and SR411, and SR389 and SR411), there were $\geq 30$ allelic differences. From a previous study, 9-29 allelic differences can be considered possibly related, whereas $>30$ are considered unrelated (11). When we compared the strains in our study to MRSA ST398 strain 4_ST398 from a hospital in Shanghai (12), we found 30-40 allelic differences between each of our 4 strains and the reference strain. There were $\geq 150$ allelic differences between the SSI strains in our study and other ST398 strains, including animal- and human-related strains, from other studies $(7,13,14)$.

All 4 strains in our study contained the same virulence factors (Table). We analyzed the lysis of human erythrocytes, which is a key determinant of $S$. aureus virulence. Erythrocyte lysis by SR389, FY20, and FY22 was stronger than for CA-MRSA HL1 $(\mathrm{p}<0.05)$ (Figure 2, panel A), whereas SR411 showed a relatively weaker lytic capacity. We used a mouse skin infection model to evaluate the virulence potential of ST398 strains in vivo. In this model, abscesses caused by SSI strains (FY20 and FY22) were significantly larger than those caused by SR411 and the HL1 agr mutant $(\mathrm{p}<0.05)$ and were similar to those caused by SR389 and HL1 ( $>0.05)$ (Figure 2, panel B). These findings demonstrated the pronounced virulence potential of SSI ST398 strains to cause invasive skin infections similar to those associated with HL1, which was a predominant CA-MRSA clone in South Korea.

\section{Conclusions}

We reported 2 ST398 MRSA strains causing SSI, which were classified into hospital-acquired MRSA according to the Centers for Disease Control and Prevention definition (15). The clinical data and cgMLST results indicated no clonal transmission in these 2 cases. These 2 patients denied livestock contact, and further genetic analysis showed characteristics of human-associated isolates. It is possible that the 2 patients were colonized with ST398 MRSA in the community and their infections developed later, after
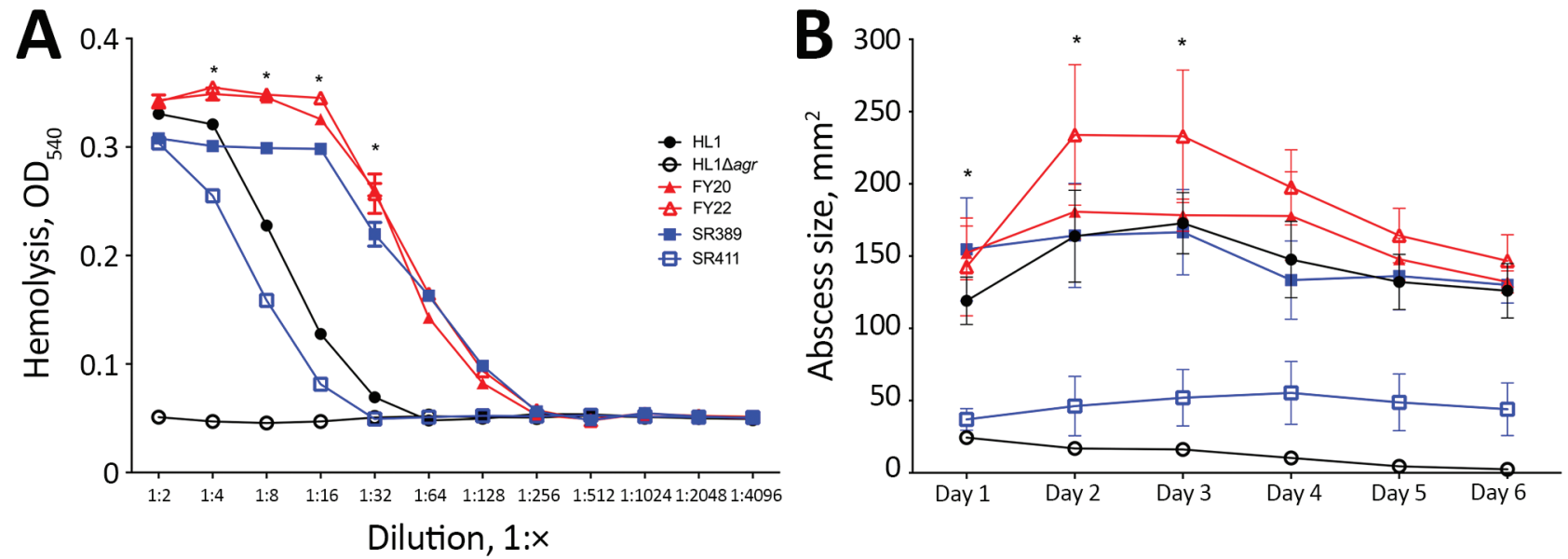

Figure 2. Virulence phenotype of MRSA ST398 isolates. A) Hemolysis analysis. The hemolytic activity of culture filtrates grown for 24 $\mathrm{h}$ was measured in triplicate at given dilutions. The mean and SE are shown. The statistical analysis used a 2-way analysis of variance between multiple groups. FY20 and FY22 were significantly stronger than other strains in dilution 1:4, 1:8, 1:16, and 1:32 ( $p<0.05)$. B) Abscess sizes in the mouse skin infection model. There were 4 mice per strain, and the mean and SE are shown. The statistical analysis used a 2-way analysis of variance to compare data for multiple groups. The abscess sizes of FY20 and FY22 were significantly larger than SR411 and the HL1 agr mutant $(p<0.05)$ but were similar to SR389 and HL1 $(p>0.05)$ at day 1, day 2, and day 3. MRSA, methicillin-resistant Staphylococcus aureus; OD, optical density; ST, sequence type. 
surgery. A limitation of this study is that we detected only 2 ST398 HA-MRSA strains, which means that it is difficult to evaluate the spreading trend and the threat of this lineage in the healthcare setting. However, our results revealed the emergence and transmission pattern of ST398 MRSA in the surgical department of a hospital in China.

\section{Acknowledgments}

We thank Min Li for providing the sequence file of the MRSA ST398 strain.

This work was supported by grants from the National Natural Science Foundation of China (grant no. 81501788) and the Zhejiang Provincial Natural Science Foundation of China (grant no. Q16H190005). All authors report no potential conflicts.

\section{About the Author}

Miss Sun is a student at Zhejiang University School of Medicine. Her research focuses on MRSA infections and using wholegenome sequencing to investigate the changing epidemiology of CA-MRSA in China.

\section{References}

1. Baker AW, Dicks KV, Durkin MJ, Weber DJ, Lewis SS, Moehring RW, et al. Epidemiology of surgical site infection in a community hospital network. Infect Control Hosp Epidemiol. 2016;37:519-26. http://dx.doi.org/10.1017/ice.2016.13

2. Verkade E, Kluytmans J. Livestock-associated Staphylococcus aureus CC398: animal reservoirs and human infections. Infect Genet Evol. 2014;21:523-30. http://dx.doi.org/10.1016/ j.meegid.2013.02.013

3. Voss A, Loeffen F, Bakker J, Klaassen C, Wulf M. Methicillinresistant Staphylococcus aureus in pig farming. Emerg Infect Dis. 2005;11:1965-6. PubMed http://dx.doi.org/10.3201/eid1112.050428

4. Huijsdens XW, van Dijke BJ, Spalburg E, van Santen-Verheuvel MG, Heck ME, Pluister GN, et al. Community-acquired MRSA and pig-farming. Ann Clin Microbiol Antimicrob. 2006;5:26. http://dx.doi.org/10.1186/1476-0711-5-26

5. Nielsen RT, Kemp M, Holm A, Skov MN, Detlefsen M, Hasman H, et al. Fatal septicemia linked to transmission of MRSA clonal complex 398 in hospital and nursing home, Denmark. Emerg Infect Dis. 2016;22:900-2. http://dx.doi.org/10.3201/eid2205.151835

6. Koyama H, Sanui M, Saga T, Harada S, Ishii Y, Tateda K, et al. A fatal infection caused by sequence type 398 methicillin-resistant Staphylococcus aureus carrying the Panton-Valentine leukocidin gene: A case report in Japan. J Infect Chemother. 2015;21:541-3. http://dx.doi.org/10.1016/j.jiac.2015.03.013

7. Golding GR, Bryden L, Levett PN, McDonald RR, Wong A, Graham MR, et al. Whole-genome sequence of livestock-associated st398 methicillin-resistant Staphylococcus aureus isolated from humans in Canada. J Bacteriol. 2012;194:6627-8. http://dx.doi.org/ 10.1128/JB.01680-12

8. Bortolami A, Williams NJ, McGowan CM, Kelly PG, Archer DC, Corrò M, et al. Environmental surveillance identifies multiple introductions of MRSA CC398 in an equine veterinary hospital in the UK, 2011-2016. Sci Rep. 2017;7:5499. http://dx.doi.org/ 10.1038/s41598-017-05559-8

9. Leopold SR, Goering RV, Witten A, Harmsen D, Mellmann A. Bacterial whole-genome sequencing revisited: portable, scalable, and standardized analysis for typing and detection of virulence and antibiotic resistance genes. J Clin Microbiol. 2014;52:2365-70. http://dx.doi.org/10.1128/JCM.00262-14

10. Chen Y, Yeh AJ, Cheung GY, Villaruz AE, Tan VY, Joo HS, et al. Basis of virulence in a Panton-Valentine leukocidin-negative communityassociated methicillin-resistant Staphylococcus aureus strain. J Infect Dis. 2015;211:472-80. http://dx.doi.org/10.1093/infdis/jiu462

11. Cunningham SA, Chia N, Jeraldo PR, Quest DJ, Johnson JA, Boxrud DJ, et al. Comparison of whole-genome sequencing methods for analysis of three methicillin-resistant Staphylococcus aureus outbreaks. J Clin Microbiol. 2017;55:1946-53. http://dx.doi.org/10.1128/JCM.00029-17

12. He L, Zheng HX, Wang Y, Le KY, Liu Q, Shang J, et al. Detection and analysis of methicillin-resistant human-adapted sequence type 398 allows insight into community-associated methicillin-resistant Staphylococcus aureus evolution. Genome Med. 2018;10:5. http://dx.doi.org/10.1186/s13073-018-0514-9

13. Schijffelen MJ, Boel CH, van Strijp JA, Fluit AC. Whole genome analysis of a livestock-associated methicillin-resistant Staphylococcus aureus ST398 isolate from a case of human endocarditis. BMC Genomics. 2010;11:376. http://dx.doi.org/ 10.1186/1471-2164-11-376

14. van der Mee-Marquet N, Corvaglia AR, Valentin AS, Hernandez D, Bertrand X, Girard M, et al. Analysis of prophages harbored by the human-adapted subpopulation of Staphylococcus aureus CC398. Infect Genet Evol. 2013;18:299-308. http://dx.doi.org/10.1016/ j.meegid.2013.06.009

15. David MZ, Daum RS. Community-associated methicillin-resistant Staphylococcus aureus: epidemiology and clinical consequences of an emerging epidemic. Clin Microbiol Rev. 2010;23:616-87. http://dx.doi.org/10.1128/CMR.00081-09

Address for correspondence: Yunsong Yu, Zhejiang University School of Medicine, Sir Run Run Shaw Hospital-Infectious Diseases, 3\# East Qingchun Rd, Hangzhou, Zhejiang 310016, China; email: yvys119@zju.edu.cn 